\title{
Sex differential marker FD for rapid sex identification of Litsea cubeba
}

Q. Wu, Y. Chen, Y. Wang and L. Lin

Institute of Subtropical Forestry, Chinese Academy of Forestry, Fuyang, Zhejiang,

China

Corresponding author: Y. Wang

E-mail:Wyd11111@126.com

Genet. Mol. Res. 14 (4): 12820-12827 (2015)

Received May 14, 2015

Accepted July 30, 2015

Published October 21, 2015

DOI http://dx.doi.org/10.4238/2015.October.21.1

ABSTRACT. Litsea cubeba is an important economic tree in China. Sex identification of the species is required to reduce breeding costs. Molecular biology is an ideal method to achieve this aim because of the lack of morphological differences between male and female plants, especially at the seedling stage. Sequence-related amplified polymorphism was used to amplify sex-related bands. Following sequencing, the amplified fragment Dwas used to create a sequencecharacterized amplified region (SCAR) marker, FD. The SCAR marker is approximately $750 \mathrm{bp}$, is female-specific, and is expected to be useful for $L$. cubeba breeding programs. Furthermore, the amplified fragment $L$ had homology to sex-determining-related genes of other species. Quantitative real-time polymerase chain reaction analysis of this fragment during flower bud development identified expression differences between male and female plants.

Key words: Sequence-related amplified polymorphism; Dioecious; Litsea cubeba; Sequence-characterized amplified region;

Sex identification; Sex-determining-related genes 


\section{INTRODUCTION}

Litsea cubeba is a dioecious perennial shrub or small tree that is widespread in areas of southern China and East Asia. It is an important economic crop in China. The peel of L. cubeba contains essential oils that have various industrial uses, and the peel is used in the manufacture of fragrances, cosmetics, and soap (Chen et al., 2013). Its seeds have long been used in the traditional Chinese medicine Cubeb. The economic value of the femaleplant is much larger than that of the male, and the ratio of female to male plants is conducive to maximal production in breeding programs (Pan et al., 2003). Presently, there is no good way to identify male and females during the seedling phenophase. This isbecause of the lack of apparent differentiation for gender discrimination in cytological or physiological features until the emergence of flowers (Chen et al., 1992). Therefore, an effective method of gender identification is necessary to improve breeding programs.

In addition to morphological or chemical methodologies, molecular technologyis often used to study sex determination (Li et al., 2013) because it is an easy, rapid, and reliable solution that is not influenced by external factors. For example, Sarmahand Sarma (2011) identified intersimple sequence repeat markersthat were linked to sex determination in Calamustenuis (Roxb.) and Khattak et al. (2006) created a genetic linkage map of Spinaciaoleracea using amplified fragment length polymorphisms and microsatellite markers to localize a sex determination locus.

We used polymerase chain reaction (PCR)-based sequence-related amplified polymorphism (SRAP) in our research because it is repeatable, concise, and has greater exon amplificationthan random amplified polymorphic DNA ( $\mathrm{Li}$ and Quiros, 2001). To facilitate SRAP, we used a forward primer that comprised 17 nucleotides with three main parts: the first 10 bases, starting at the $5^{\prime}$ end, are "filler" sequence with no specific constitution; this is followed by a CCGG sequence, which increases the probability of exon amplification; and the final part consists of three selective nucleotides at the $3^{\prime}$ end. The reverse primer was 18 nucleotides long and used an 11-nucleotide filler sequence followed by an AATT sequence and three selective nucleotides at the 3 ' end.

Sequence-characterized amplified region (SCAR) is a PCR-based molecular marker technology that is simpler, faster, more stable, and more practical for use in breeding programs than SRAP. The primers that were used for the SCAR markers were the same as those described by Horejsi et al. (1999). The specific fragments that were identified from SRAPwere sequenced andused to create SCAR markers. When thisprocess is successful, it allows for easy determination of differences in band size using agarose gel electrophoresis (Rao et al., 2012).

The sex determination mechanisms of dioecious plants are largely unknown. There are few reports on the topic of sex chromosomes in dioecious species (Ming et al., 2007), and, currently, there is no report of sex chromosomes in L. cubeba. This study of L. cubeba male and female sex determination mechanisms lays the foundation for future breeding programs and biological research.

\section{MATERIAL AND METHODS}

\section{Plant material}

Samples from six flower bud developmental stages were collected from five female and five male L. cubeba plants from the Fuyang Forest Park, Zhejiang Province, China. The distance between each tree was greater than $50 \mathrm{~m}$. Trees were at least three years old, and their sex was 
determined from their flowers. Samples were collected on six dates in2013: February 20, February 27, March 2, March 7, March 14, and March 23, by which time the male flowers had withered. The samples were placed on ice during collection and stored at $-80^{\circ} \mathrm{C}$ until required. Other buds were collected from four female and four male $L$. cubeba plants from four typical $L$. cubeba natural populations (Jian'ou, Fujian Province; ZhiJin, Guizhou Province; Bijie, Guizhou Province; and Xinning, Hunan Province) (Chen et al., 2013).

\section{DNA isolation}

Genomic DNA was isolated from approximately $100 \mathrm{mg}$ frozen buds starting from February 20, by using a DN15 Plant Genomic DNA kit protocol (Aidlab Biotechnologies Co., Ltd., China) according to the manufacturer protocol, and then the DNA was dissolved in deionized water. DNA concentrations were determined using a Q5000 UV-V is spectrophotometer (Quawell Technology, USA). DNA was divided into a male DNA pool, which contained equal quantities from the five individual male plants, and a female DNA pool, which contained equal quantities from the five individual female plants, in accordance with the bulk segregant analysis procedure (Michelmore et al., 1991; Almeida et al., 2013). The DNA pools were diluted to a concentration of $50 \mathrm{ng} / \mu \mathrm{L}$ for use in agarose gel electrophoresisand were stored at $-20^{\circ} \mathrm{C}$ until required.

\section{SRAP marker system protocol}

SRAP marker design was based on that described by Li and Quiros (2001). A total of 25 primers were designed: nine forward and 16 reverse primers. The primers were synthesized by SangonBiotech (China). PCR temperature conditions were determined using temperature gradient PCR. The thermal profile was as follows: initial denaturation at $94^{\circ} \mathrm{C}$ for $5 \mathrm{~min}$; five cycles of denaturation at $94^{\circ} \mathrm{C}, 1 \mathrm{~min}$ annealing at $35^{\circ}-45^{\circ} \mathrm{C}$, and $1 \mathrm{~min}$ extension at $72^{\circ} \mathrm{C}$; the annealing temperature was then raised to $50^{\circ} \mathrm{C}$ for a further $35 \mathrm{cycles}$; and the final extension was performed at $72^{\circ} \mathrm{C}$ for $5 \mathrm{~min}$. We determined $37^{\circ} \mathrm{C}$ to be the optimal annealing temperature for the first five cycles, and used this temperature condition for subsequent amplifications.

Orthogonal design direct analysis was used for PCR optimization (He et al., 1998). We chose template, primers, dNTP, and buffer (contains $\mathrm{Mg}^{2+}$ ) concentrations as optimization factors, and each factor had four different gradients. Takara Ex Taq, 10X Ex Taq Buffer ( $\left.\mathrm{Mg}^{2+} \mathrm{Plus}\right)$, and dNTP Mixture were supplied by Takara (China). Sixteen different PCR products were determined by $2 \%(\mathrm{w} / \mathrm{v})$ agarose gel electrophoresis in accordance with the L16 $\left(4^{4}\right)$ orthogonal table. Following calculations, the strength and number of bands were scored from 1 to 16 . We selected the following conditions for the final PCR system: system volume of $20 \mu \mathrm{L}, 140 \mathrm{ng}$ DNA template, $13 \mathrm{ng}$ primer, $2 \mu \mathrm{L}$ dNTP mixture, $2 \mu \mathrm{L}$ buffer, $0.1 \mu \mathrm{L}$ Extaq, and $10.5 \mu \mathrm{L}$ water. The nine forward primers and 16 reverse primers allowed for 144 distinct PCRs.

\section{TA cloning and sequencing}

All PCR products were run on a $2 \%(\mathrm{w} / \mathrm{v})$ agarose gel. Specific bands were spliced under an ultraviolet (UV) lamp and purified using an AxyPrep DNA gel extraction kitfollowing manufacturer instructions (Axygen, USA).Purified DNA was cloned into a pMD18-T vector according to the manufacturer instructions (Takara). A minimum of three clones for each band were sequenced. 


\section{SCAR design and analysis}

Specific fragment sequences were analyzed using DNAMAN5.0 (LynnonBiosoft, USA). Primers were designed using Primer3 web (http://primer3.wi.mit.edu/) with the aim to clone the sequences of both male and female genomic DNA. Primers were synthesized by Sangon. The following PCR conditions were used: $95^{\circ} \mathrm{C}$ for $10 \mathrm{~min} ; 35$ cycles of $95^{\circ} \mathrm{C}$ for $30 \mathrm{~s}, 52^{\circ} \mathrm{C}$ for $40 \mathrm{~s}$, and $72^{\circ} \mathrm{C}$ for $1 \mathrm{~min}$; and $72^{\circ} \mathrm{C}$ for $7 \mathrm{~min}$. Amplifications were made using GoldStar Taq MasterMix (Beijing CoWin Biotech, China). All PCR products were resolved on a $1 \%(\mathrm{w} / \mathrm{v})$ agarose gel, and the extracted bands were sent to Sangon for sequencing.

\section{RNA isolation and first strand cDNA synthesis}

Two female and two male plants were selected as the material for RNA extraction. The RNA was isolated from approximately $100 \mathrm{mg}$ frozen buds or flowers from six developmental stages using the RN38 EASYsp in plus Plant RNA kit in accordance with the manufacturer instructions (Aidlab Biotech). Purified RNA was quantified using a Q5000 UV-Vis spectrophotometer, and the concentration and integritywere checked by $1 \%(\mathrm{w} / \mathrm{v})$ agarose gel electrophoresis. Approximately $3 \mu \mathrm{g}$ total RNA was used for cDNA synthesis using a PrimeScript 1st Strand cDNA Synthesis Kit (Takara) following the manufacturer instructions. The CDNA was then diluted 1:20 with nucleasefree water for use in quantitative real-time PCR (qRT-PCR).

\section{qRT-PCR}

qRT-PCR primers were designed using Primer3 web (http://primer3.wi.mit.edu/). qRT-PCR was performed in 96-well plates using a 7300 Real Time PCR System (ABI PRISM, USA) and a SYBR Premix Ex Taq Kit (Takara) in accordance withthe manufacturer instructions. Reactions were performed in a $20 \mu \mathrm{L}$ system as follows: $10 \mu \mathrm{L}$ SYBR Premix Ex Taq, $0.4 \mu \mathrm{L} 10 \mu \mathrm{M}$ PCR forward primer, $0.4 \mu \mathrm{L} 10 \mu \mathrm{M}$ PCR reverse primer, $0.4 \mu \mathrm{L}$ 50X ROX reference Dye, $2 \mu \mathrm{L} \mathrm{cDNA}$, and $6.8 \mu \mathrm{L}$ $\mathrm{dH}_{2} \mathrm{O}$. The cycling conditions were $30 \mathrm{~s}$ at $95^{\circ} \mathrm{C}$ and 40 cycles of $95^{\circ} \mathrm{C}$ for $5 \mathrm{~s}$ and $60^{\circ} \mathrm{C}$ for $31 \mathrm{~s}$. Melting curve analysis was performed to verify the specificity of amplicons after 40 PCR cycles.

\section{RESULTS}

\section{SRAP analysis}

Of the 144 primer pairs that were used, 101 could amplify normal band fragments. The number of bands observed was 1251 in males and 1256 in females. Thirty-three pairs of primers exhibited differences between males and females, and there were 17 male-specific and 22 femalespecific bands. Eleven of these were selected for further analyses based on their visibility and stability. The 11 bands, which were labeled A, B, D, E, F, G, H, J, KL, M, and Y, were amplified from the primer combinations Me2 and Em11, Me1 and Em11, Me1 and Em11, Me1 and Em14, Me2 and Em14, Me2 and Em14, Me2 and Em11, Me1 and Em15, Me2 and Em7, Me3 and Em13, and Me2 and Em14, respectively (Figure 1, Table 1).

\section{SCAR analysis}

Twelve amplification fragments were sequenced (n.b., the extra band was a result of KL 
being identified as two distinct fragments, $\mathrm{K}$ and $\mathrm{L}$ ), and SCAR primers were designed to amplify the male and female DNA of the 12 sequences. Primers DOf and DOr (Table 1), which were based on sequence $D$, were used to amplify the fragment FD from the male and female genome. The FD fragment was found only in the female genome (Figure 2).

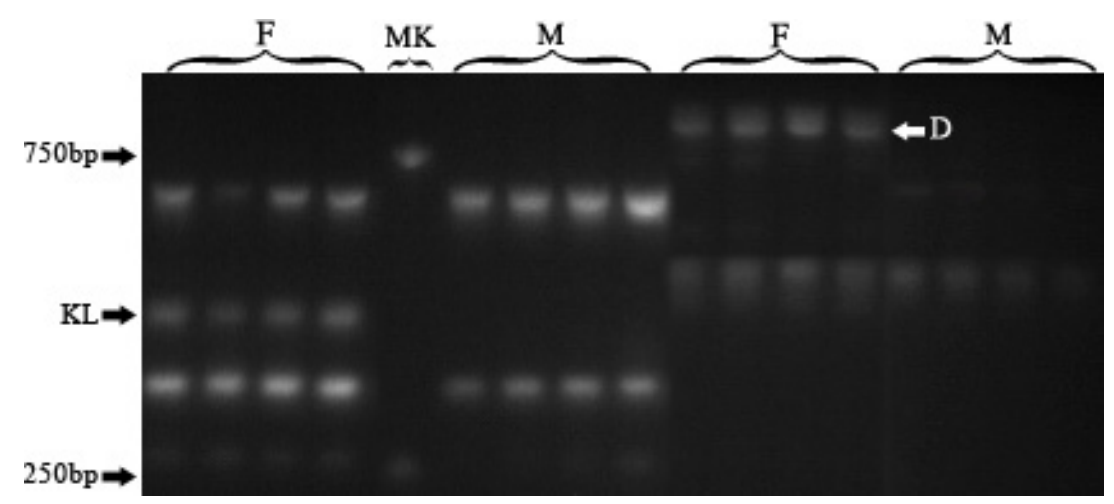

Figure 1. Examples of specific amplification products used for SRAP analysis of the male and female DNA pools. Male and female DNA pools were amplified using the primers ME1 and EM11 for D, and ME2 and EM7 for KL. The arrows indicate the position of the male-specific amplification product $K L$, and the female-specific fragment $D . K e y: M=M a l e$. $\mathrm{F}=$ Female. $\mathrm{Mk}=2000 \mathrm{bp}$ marker.

Table 1. List of primers used in this study.

\begin{tabular}{ll}
\hline SRAP Forward primer & \\
ME1 & 5'-TGAGTCCAAACCGGATA-3' \\
ME2 & 5'-TGAGTCCAAACCGGAGC-3' \\
ME3 & 5'-TGAGTCCAAACCGGAAT-3' \\
SRAP Reverse primer & \\
EM7 & 5'-GACTGCGTACGAATTATG-3' \\
EM11 & 5'-GACTGCGTACGAATTTCG-3' \\
EM13 & 5'-GACTGCGTACGAATTGGT-3' \\
EM14 & 5'-GACTGCGTACGAATTCAG-3' \\
EM15 & 5'-GACTGCGTACGAATTCTG-3' \\
SCAR Forward primer & \\
D0f & 5'-TGACACTCCTTACTACTCGGG-3' \\
D2f & 5'-TTGTCCACCGGCATAGAGAT-3' \\
D3f & 5'-GGAGATGAGGAAGATGATGGC-3' \\
SCAR Reverse primer & \\
D0r & 5'-GCAAACCATGGTCCTAAGCC-3' \\
D1r & 5'-ACCATGGTCCTAAGCCAACT-3' \\
D2r & 5'-GACCCACTGCCATTTCAACA-3' \\
\hline
\end{tabular}

To investigate this potential marker further, we designed four primers according to the sequence of fragment D (Table 1). This resulted in six primer pairs (when including Dof and D0r) that could be used to verify the differences between the male and female DNA pools (Figure 2). Amplified fragment $D$ is 787 nucleotides long, and we speculate that the sequence differences between the male and female occurs in the 539-732 sequence range.

Next, eight individual male and female plants from Fuyang and the four L. cubeba populations were screened to verify the use of the SCAR primers DOf and DOr. Figure 3 shows that the SCAR primers could be used to identify sex types in different $L$. cubeba populations. 


\section{Analysis of gene expression}

An alignment of the 12 sequences using the National Center for Biotechnology Information database (http://www.ncbi.nlm.nih.gov/) revealed that sequence fragment $L$ may be relevant to sex determination because it has $79 \%$ identity to the mRNA of the Vitis vinifera sex determination protein tasselseed-2-like (LOC100265336). The sequence of fragment $L$ is as follows: 5'-CGCGTCAT GGTCCAAAGGGGATGGGCTGCATCATCTCAACCGCTAGTGTAGCAGGAGTCATGGGAGGGC TTGGTCCCCATGCATACACTGCCTCCAAGCATGCAATTGTGGGGCTCACGAAGAACGCGGC GTGTGAGCTTGGAAGGGCAGGGATTAGAGTGAATTGTATCTCTCCCTTTGGGGTGGCAACAC CTATGCTTGTTAATGCATGGAGAGGGGGTGAGGAGGAGGAAGATGACTGTATTGGATTGAGT ATTCCTAGTGAACAAGAAGTTGAAAATATGGAGGAGATTGTG-3'.

In numerous studies carried out in our laboratory, the ubiquitin-conjugating enzyme (UBC) gene has proven to be a stable reference gene for investigations of $L$. cubeba expression. Therefore, we used UBC as a reference gene for qRT-PCR experiments that aimed to verify differences in fragment $L$ expression in male and female plants at six flower bud developmental stages. Figure 4 shows that the expression of amplification fragment $L$ increases in the male during the flower bud development process. In female plants, the expression of $L$ is low and changes little during flower bud development. We speculate thatamplified fragment $L$ may be related to the development of male plants or flowers.

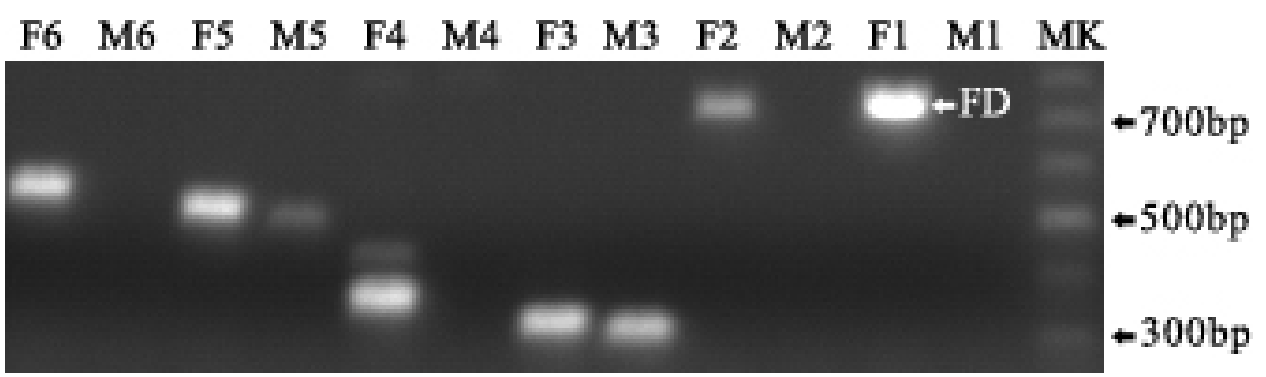

Figure 2. Amplification of fragment $D$ using SCAR primers. The letters $F$ and $M$ represent the female pool and male pool, respectively; numbers 1-6 represent the primers D0f and D0r, D0f and D1r, D2f and D2r, D3f and D2r, D0f and $\mathrm{D} 2 \mathrm{r}, \mathrm{D} 2 \mathrm{f}$ and D1r. Fragment $\mathrm{D}$ has 787 nucleotides. The range of amplification covered by primer 1 is 17-732; primer 2 is 18-728; primer 3 is 206-539; primer 4 is 355-728; primer 5 is $17-539$; and primer 6 is 206-728. Mk $=100$ bp marker.

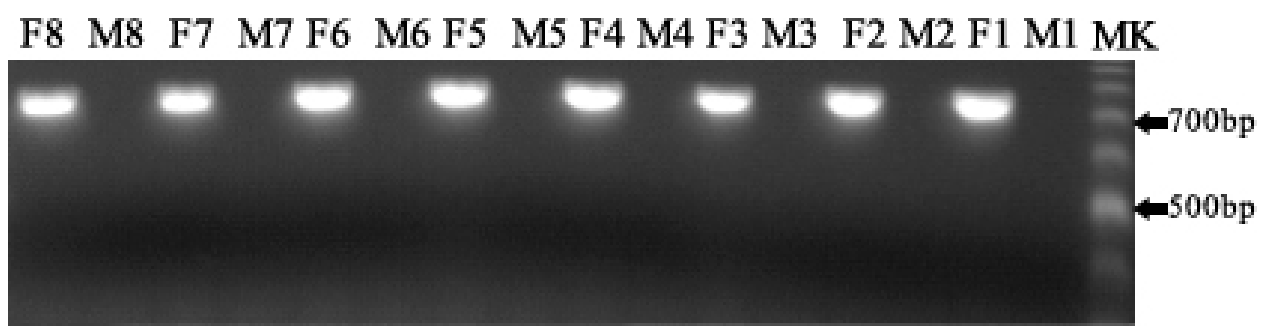

Figure 3. Testing of SCAR marker primers on individual plants from five L. cubeba populations. The letters $F$ and $M$ represent individual female and male plants, respectively, from different $L$. cubeba populations; the numbers 1-4 represent plants from the Fuyang population; numbers 5-8 represent samples from Jian'ou, Fujian Province; ZhiJin, Guizhou Province; Bijie, Guizhou Province; and Xinning, Hunan Province, respectively. Mk = 100 bp marker. 


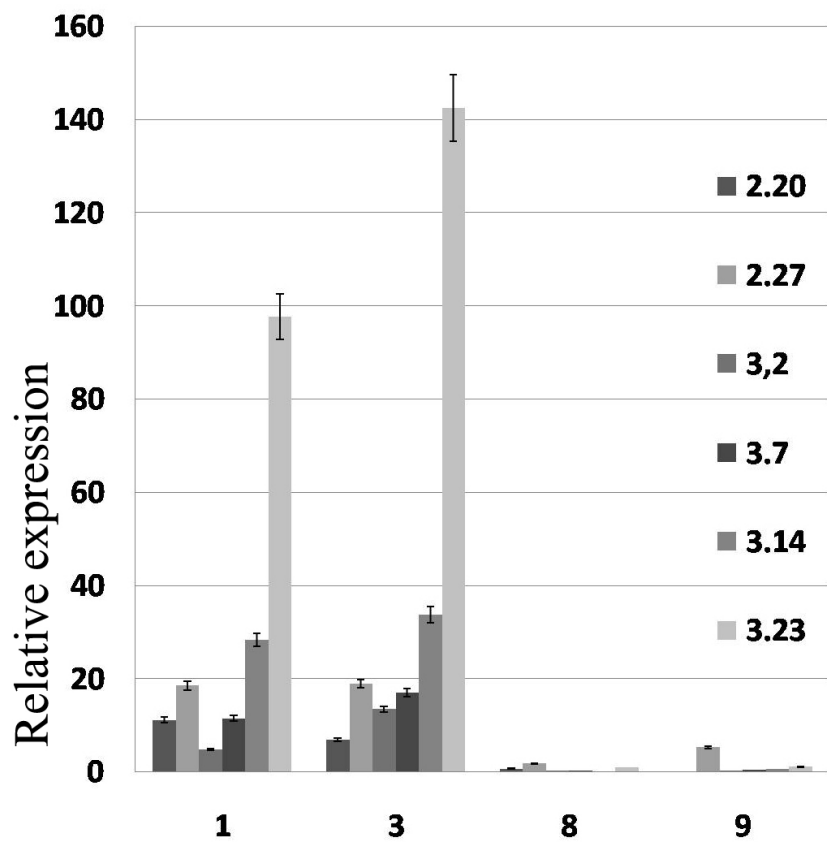

Figure 4. Differences in expression levels of fragment $L$ in male and female plants during six distinct flower bud developmental stages. qRT-PCR was used to measure the expression levels of two male plants (1, and 3 ), and two female plants (8, and 9). The different bars represent different developmental collection periods in 2013 as follows: 20 February; 27 February; 2 March; 7 March; 14 March; and 23 March. The vertical axis represents relative expression. All expression levels are relative to the reference gene UBC.

\section{DISCUSSION}

According to recent studies, there are three possible sex-determining mechanism models in spermatophytes. These involve heteromorphic sex chromosomes, single major gene control of sex determination (Ming et al., 2007), and hormonal regulation (Dellaporta and Calderon-Urrea, 1993).

The mechanism of sex determination is still unknown in $L$. cubeba. This is a consequence ofthe lack of a complete genome sequence andthe lack of prior investigations of sex chromosomes in this species. This study identified 17 male-specific and 22 female-specific amplified fragments from a total of 2507 amplified fragment bands. Of the 12 bands that we subjected to further analyses, only onehad aspecific difference in sequence between the male and female. This band showed differences in a sequence of a few hundred nucleotides. Our findings suggest that there are few differences between males and females in the $L$. cubeba genome, and we speculate that the sex determination of $L$. cubeba may be controlled by a single locus or through hormonal regulation. It is also possible that the sex chromosomes of $L$. cubeba occurred early in its evolution, leading to very few differences between the male and female (Grant et al., 1994). Future work is required to determine further differences betweenmale and female $L$. cubeba karyotypes.

In this study, we identified sexual differences using SRAP fragments, and then we created SCAR markers based on those fragment sequences. We verified that the SCAR markers were stable in several distinct populations of $L$. cubeba. This demonstrated that the SCAR marker could be used for sex determination of $L$. cubeba. The use of the DOf and DOr primer pair can reliably 
beused for sex identification, and this is expected to save time and reduce costs in breeding programs.

Furthermore, we identified a gender-related sequence that had differential expression during the flower bud development process. The expression level of this fragment increased in themale buds and flowers during the developmental process, but it remained low in female plants. We are currently unable to definitively conclude that this sequence is related to male flower development. Therefore, future work is required to map andclone the full-length gene associated with this fragment and to analyze its expression in the stem and root during flower bud development.

\title{
ACKNOWLEDGMENTS
}

\author{
Research financially supported by the National Natural Science Foundation of China.
}

\section{REFERENCES}

Almeida IG, lanella P, Faria MT, Paiva SR, et al. (2013). Bulked segregant analysis of the pirarucu (Arapaima gigas) genome for identification of sex-specific molecular markers. Genet. Mol. Res. 12: 6299-6308.

Chen $Y$ and Hu Z (1992). Litseacubeba early identification of male and female plants. China Forest. Sci. Technol. 1: 27-28.

Chen Y, Wang Y, Han X, Si L, et al. (2013). Biology and chemistry of Litseacubeba, a promising industrial tree in China. J. Essent. Oil Res. 25: 103-111.

Dellaporta SL and Calderon-Urrea A (1993). Sex determination in flowering plants. Plant Cell 5: 1241-1251.

Grant S, Hunkirchen B and Saedler H (1994). Developmental differences between male and female flowers in the dioecious plant Silenelatifolia. Plant J. 6: 471-480.

He Z, Liu Y, Chen L, Cao M, et al. (1998). Orthogonal design-direct analysis for PCR optimization. Hunan Yi Ke Da Xue Xue Bao 23: 403-404.

Horejsi T, Box JM and Staub JE (1999). Efficiency of randomly amplified polymorphic DNA to sequence characterized amplified region marker conversion and their comparative polymerase chain reaction sensitivity in cucumber. J. Am. Soc. Hortic. Sci. 124: 128-135.

Khattak JZK, Torp AM and Andersen SB (2006). A genetic linkage map of Spinaciaoleracea and localization of a sex determination locus. Euphytica 148: 311-318.

Li G and Quiros CF (2001). Sequence-related amplified polymorphism (SRAP), a new marker system based on a simple PCR reaction: its application to mapping and gene tagging in Brassica. Theor. Appl. Genet. 103: 455-461.

Li YX, Wang XG, Yang CH, Cong LL, et al. (2013). Identification of a locus characteristic of male individuals of buffalo grass [Buchloe dactyloides (Nutt.) Engelm.] by using an RAPD marker. Genet. Mol. Res. 12: 4070-4077.

Michelmore RW, Paran I and Kesseli RV (1991). Identification of markers linked to disease-resistance genes by bulked segregant analysis: a rapid method to detect markers in specific genomic regions by using segregating populations. Proc. Natl. Acad. Sci. U. S. A. 88: 9828-9832.

Ming R, Wang J, Moore PH and Paterson AH (2007).Sex chromosomes in flowering plants. Am. J. Bot. 94: 141-150.

Pan XJ, Chen WJ and Hou HB (2003). Current processing and development of Litseacubeba. Econ. Forest Res. $21: 79-80$.

Rao HO, Deng JC, Wang WM and Gao ZX (2012). An AFLP-based approach for the identification of sex-linked markers in blunt snout bream, Megalobrama amblycephala (Cyprinidae). Genet. Mol. Res. 11:1027-1031.

Sarmah P and Sarma RN (2011). Identification of a DNA marker linked to sex determination in Calamus tenuis Roxb., an economically important rattan species in northeast India. Mol. Breed. 27: 115-118. 